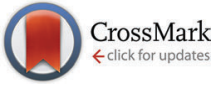

Cite this: Chem. Commun., 2015, 51, 11697

Received 27th April 2015, Accepted 16th June 2015

DOI: $10.1039 / c 5 c c 03507 d$

www.rsc.org/chemcomm

\section{Surface plasmon $\&$ visible light for polymer functionalization of mesopores and manipulation of ionic permselectivity $\dagger$}

\author{
Nicole Herzog, ${ }^{a}$ Jonas Kind, ${ }^{a}$ Christian Hess ${ }^{b}$ and Annette Andrieu-Brunsen*a
}

\begin{abstract}
The near-field of surface plasmons is locally confined to nanometer dimensions. Here, we use surface plasmons to initiate polymerfunctionalization of mesoporous silica films allowing subsequent ionic permselectivity gating based on polymer charge. We expect this functionalization approach to open a new dimension of functional miniaturization e.g. in nanofluidics.
\end{abstract}

Many efforts are concentrated on the fabrication of miniaturized structures and building of nanodevices. To miniaturize devices and functionalization the limits of synthetic protocols have to be adapted to confine synthesis to the nanoscale. Spatiotemporal control over a polymerization reaction is mainly offered by photoinitiation procedures. ${ }^{1}$ Recently, even sunlight was proposed to be used for a controlled polymerization. ${ }^{2}$ Using conventional photolithography, local resolution is limited by Abbe's law of diffraction. ${ }^{3}$ On the other hand, the electromagnetic field of nearfield modes is characterized by local nanometer dimensions and tunable wavelength in the visible. Surprisingly, surface plasmons are mainly used for sensing ${ }^{4,5}$ and only few studies on surfaceplasmon induced polymerization are reported. ${ }^{6,7}$ Polymerization in the visible can for example, be achieved with multicomponent initiating systems, such as the combination of dyes as photosensitizer and amines as radical initiating molecules. ${ }^{8}$ Examples are cyanine dyes, ${ }^{9,10}$ methylene blue, ${ }^{11}$ or alexa dyes. ${ }^{12-14}$ Combining such visible-light photo-initiation concepts with mesoporous membranes (surface) functionalization of mesopores, visible light-initiated, localized near-field functionalization in water becomes accessible. ${ }^{15}$ Very recently the group of Soppera ${ }^{6,16}$ as well as the group of Kreiter ${ }^{7,17}$ demonstrated the possibility to initiate a solution photopolymerization with the surface plasmon of metallic nanoparticles. In this context Chegel and co-workers used surface plasmon at a planar metal interface to perform

${ }^{a}$ Technische Universität Darmstadt, Ernst-Berl-Institut für Technische und Makromolekulare Chemie, Alarich-Weiss-Str. 4, D-64287 Darmstadt, Germany. E-mail: brunsen@cellulose.tu-darmstadt.de

${ }^{b}$ Technische Universität Darmstadt, Eduard-Zintl-Institut für Anorganische und Physikalische Chemie, Alarich-Weiss-Str. 8, D-64287 Darmstadt, Germany

$\dagger$ Electronic supplementary information (ESI) available. See DOI: 10.1039/c5cc03507d photochemistry and to in situ generate a biosensor matrix without taking care about surface immobilization. ${ }^{11}$ So far these experiments are focused on generating a relatively undefined polymer matrix and they are not taking into account the third dimension perpendicular to a surface or covalent surface grafting of the generated polymer. Besides, polymer-functionalized mesoporous thin films are known to be able to gate ionic permselectivity by excluding ions of identical charge and pre-concentrating countercharged ions. ${ }^{18,19}$ Such functionalized membranes are highly interesting for nanofluidics, filtration-sensing elements, sensor arrays. ${ }^{20}$

The applied concept of visible light induced surface polymer functionalization of mesoporous thin silica films is summarized in Fig. 1.

Three different dyes with absorption wavelengths of $390 \mathrm{~nm}$ (2-chlorothioxanthone), $510 \mathrm{~nm}\left(4^{\prime}, 5^{\prime}\right.$-dibromofluorescein), and $660 \mathrm{~nm}$ (methylene blue) in combination with bis-(2-hydroxyethyl)3 -aminopropyltriethoxysilane as co-initiator ${ }^{6,21}$ are used for visible

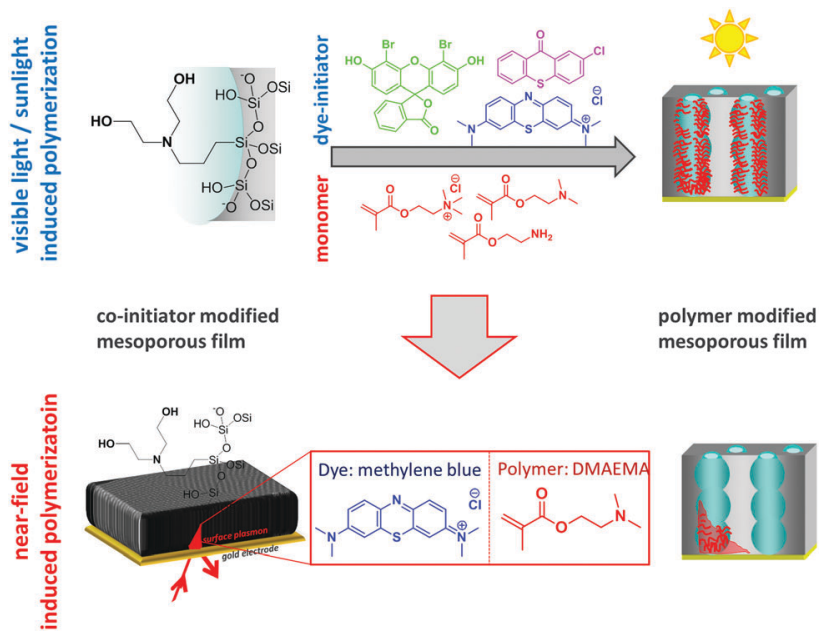

Fig. 1 Schematic presentation of the visible light, sunlight, or surface plasmon near-field induced surface polymer modification of mesoporous thin silica films using dye-sensitized polymerizations. 


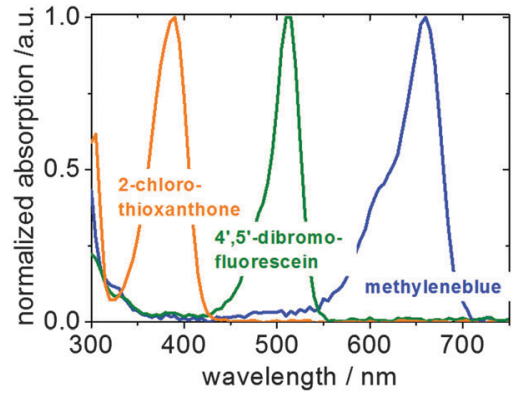

Fig. 2 UV-vis spectra of the applied dye initiators 2-chlorothioxanthone (orange), 4',5'-dibromofluorescein (green), and methylene blue (blue) measured in a water-based solution.

light initiated photopolymerization. The absorption spectra (Fig. 2) clearly show that the absorption maxima are well separated, covering a wavelength range from 400 to almost $700 \mathrm{~nm}$. This allows to individually address three different wavelengths for synthetic modifications.

To ensure covalent polymer attachment to the mesopore wall we covalently grafted the co-initiator 3-[bis(2-hydroxyethyl)amino]propyl-triethoxysilane to the mesoporous silica thin films. After silica surface modification with the co-initiator, XPS analysis (Fig. 3) revealed one co-initiator molecule per approximately ten silica atoms in case of functionalization at an elevated temperature of $80{ }^{\circ} \mathrm{C}$ for 225 minutes. Reducing the reaction time to 45 minutes resulted in a slightly decreased co-initiator concentration of one molecule per 12.5 silica atoms (Fig. S3, ESI $\dagger$ ) as extracted from integration of the N1s signals at around $400 \mathrm{eV}$.

Subsequently, the mesoporous silica films were functionalized with polymer using 2-aminoethylmethacrylate (AEMA), 2-(dimethylamino)ethylmethacrylate (DMAEMA) or [2-(methacryloyloxy)ethyl]trimethylammonium chloride (METAC) by irradiation with visible light, sunlight or surface-plasmon near field modes in the presence of the initiating dye. Experimental details can be found in the ESI. $\dagger$

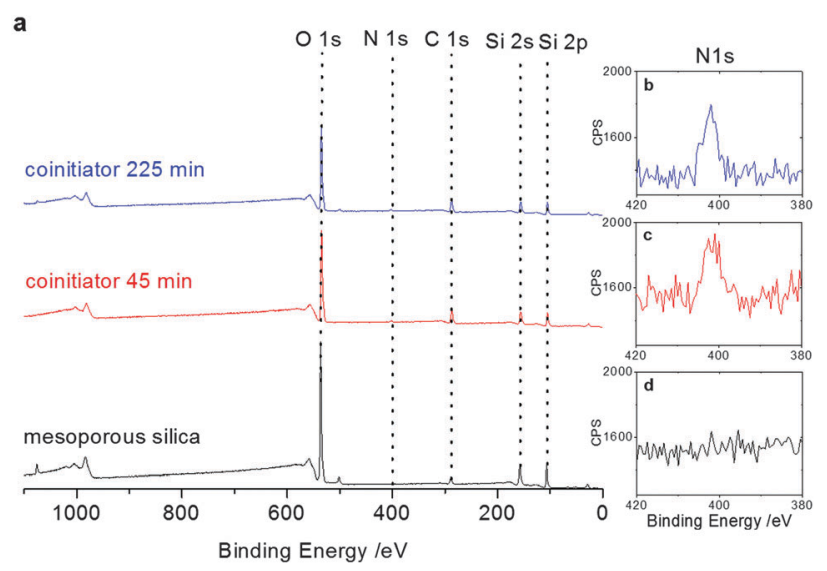

Fig. 3 (a) XP-spectra showing the co-initiator presence after functionalization for 45 minutes (red) and 225 minutes (blue) in comparison to non-functionalized mesoporous thin films (black) including the respective zoom into the N1s signal for the untreated mesoporous silica film (d) as well as after functionalization for 45 minutes (c) and 225 minutes (b).

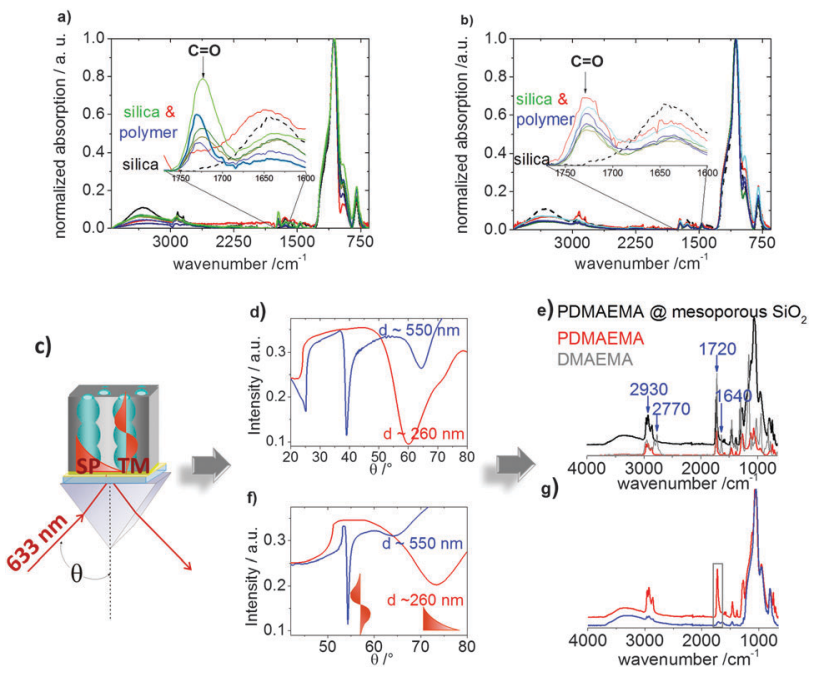

Fig. 4 Infrared spectra showing the generation of polymer as detected by the $\mathrm{C}=\mathrm{O}$ vibration at $1725 \mathrm{~cm}^{-1}$ for the polymerization of (a) METAC with methylene blue (red), DMAEMA with 4',5'-dibromofluorescein (cyan), methylene blue (blue), 2-chlorothioxanthone (marine) and AEMA with $4^{\prime}, 5^{\prime}$-dibromofluorescein (olive), methylene blue (dark yellow), 2-chlorothioxanthone (green) for non-plasma treated samples and (b) mesoporous silica films after plasma treatment and thus destruction of the co-initiator at the external mesoporous film surface. (c) Schematic view of the near-field induced polymerization in the Kretschmann configuration. The angular spectra of a $\sim 260 \mathrm{~nm}$ (red) and a $\sim 550 \mathrm{~nm}$ (blue) thick mesoporous film are shown in the presence of air (d) and monomer solution (f). (e) Infrared spectra for the polymer PDMAEMA (red) and the PDMAEMA modified mesoporous $\sim 260 \mathrm{~nm}$ film (black) in comparison to the DMAEMA monomer (grey). In case of reduced laser intensity, shorter reaction time and an angle of almost no energy coupling $\left(64^{\circ}\right)$ in case of the $\sim 550 \mathrm{~nm}$ film only a very low amount of polymer is generated ( $g$, blue).

Initially, polymer functionalization using an external irradiation source in the presence of different monomers and dyes is discussed. Polymer functionalization is monitored by following the polymer $\mathrm{C}=\mathrm{O}$ infrared absorption at $1725 \mathrm{~cm}^{-1}$ (Fig. 4a). All infrared spectra show the characteristic bands of the inorganic framework and the organic functions incorporated into the mesoporous films after extraction of unbound monomer and polymer. ${ }^{22}$ All spectra are normalized to the silica matrix using the transverse optical Si-O-Si modes at $1065 \mathrm{~cm}^{-1}\left(\nu_{\text {as }}\right)$. Especially, the remaining $\mathrm{C}=\mathrm{O}$ stretching at $1725 \mathrm{~cm}^{-1}$ after extensive extraction indicates the successful visible-light induced polymerization at the mesoporous silica thin film. To proof that polymerization occurs as well inside the mesopores and not primarily on the external film surface, a short $\mathrm{CO}_{2}$ plasma treatment was used to destroy the co-initiator located at the external mesoporous film surface. ${ }^{22}$ Subsequent polymerization as well shows polymer functionalization with only slightly reduced $\mathrm{C}=\mathrm{O}$ infrared absorption at $1725 \mathrm{~cm}^{-1}$ (Fig. 4b) indicating the polymer functionalization of the mesopore walls.

Comparing the IR absorption at $1725 \mathrm{~cm}^{-1}(\mathrm{C}=\mathrm{O})$ for METAC and AEMA with plasma treatment (Fig. 4b) and without plasma treatment (Fig. 4a) a slightly reduced absorption mostly between $10-20 \%$ and up to $50 \%$ in case of METAC polymerization with methylene blue is observed. This indicates a reduction in polymer amount relative to the silica amount for plasma-treated 
films as compared to non-plasma treated samples. The observed reduction in $\mathrm{C}=\mathrm{O}$ absorption is not proportional to the inner versus outer surface ratio which might indicate diffusional limitations for the polymerization in mesopores under the applied experimental conditions. Nevertheless, a significant amount of polymer is generated within the mesopores (Fig. 4b) showing the diffusion of dye and monomer into the mesoporous film and the potential of visible light induced polymerization as functionalization strategy for the inner mesopore surface. Polymerization inside the mesopores was additionally verified by ellipsometry measurements. The film thickness was determined to be around $260 \mathrm{~nm}$ after polymerization and the refractive index increased upon each modification step from 1.26 to 1.36 after co-initiator modification, exceeding 1.40 up to 1.46 after polymerization. Based on the effective medium approximation ${ }^{23}$ a total pore filling of $30-45 \%$ in case of METAC polymerization initiated with methylene blue is detected. Analyzing the solution polymer for of DMAEMA polymerization by GPC measurements, a $M_{\mathrm{w}}$ between $1 \times 10^{5}$ and $7 \times 10^{5}$ with a PDI between 2.7 and 5.7 was observed after initiation with $4^{\prime}, 5^{\prime}$-dibromofluorescein or 2-chlorothioxanthone. In case of polymerization at planar silicon wafer substrates the solution polymer molecular weight according to GPC measurements was smaller $\left(3-8 \times 10^{4} \mathrm{~g} \mathrm{~mol}^{-1}\right)$. The exact values are summarized in Table S2 (ESI $\dagger$ ). For surface polymer analysis an exemplary degrafting for PDMAEMA initiated with methylene blue and with 2-chlorothioxanthone revealed very small amounts of polymer and molecular weights of 5 monomer units per chain as determined by mass spectroscopy. This supports earlier observations on polymerization in confinement of mesopores resulting in lower polymer molecular weight as compared to polymerization at planar surfaces and in solution. ${ }^{22,24}$ Further details can be found in the ESI. $\dagger$

Ellipsometry, infrared spectroscopy, and GPC analysis of the solution polymer indicate a successful polymerization initiated by visible light between 400 and $700 \mathrm{~nm}$ for three different initiators and three different monomers METAC, AEMA, DMAEMA. This is building the basis for localized polymerization initiated by near field modes such as surface plasmons. Even sunlight exposure resulted in polymerization as verified by infrared spectroscopy. In the following, the above discussed polymerization conditions are transferred to surface plasmon-initiated surface polymer functionalization of a mesoporous silica film supported on a thin gold film.

Under the applied experimental conditions surface plasmons are induced between 400-700 $\mathrm{nm}$ in the Kretschmann configuration (Fig. 4c). Surface plasmons generated in a $50 \mathrm{~nm}$ gold film with a He-Ne-laser excitation at $633 \mathrm{~nm}$ in the Kretschmann configuration ${ }^{25}$ are characterized by an exponentially decaying electromagnetic field perpendicular to the metal surface with a penetration depth of $\sim 200 \mathrm{~nm} .{ }^{25}$ We functionalized mesoporous silica films by using the highly localized surface plasmon electromagnetic field to initiate dye-sensitized polymerization at $633 \mathrm{~nm}$. At this wavelength methylene blue as initiating dye is used.

Mesoporous silica thin films were deposited on a goldcovered $(50 \mathrm{~nm})$ high refractive index LaSFN9 glass substrate.
The gold layer is covered with a few $\mathrm{nm}$ thick, sputtered $\mathrm{SiO}_{x}$ layer to facilitate mesoporous film deposition by dip coating and evaporation induced self-assembly (EISA). ${ }^{26}$ Irradiation with a He-Ne-laser in the Kretschmann configuration results in generation of a surface plasmon under resonant angle (Fig. 4d and f). The resonant angle for the mesoporous silica film deposited on the $\mathrm{SiO}_{x}$-covered gold interface is around $60-65^{\circ}$ in the presence of air and shifts to up to $75^{\circ}$ upon addition of water-based monomer solution (Fig. $4 \mathrm{~d}$ and f). The mesoporous film thickness was determined to be around $260 \mathrm{~nm}$ for a one layer mesoporous film and around $550 \mathrm{~nm}$ for a two layer mesoporous film, which is in accordance with ellipsometry measured on wafer substrates. Surface plasmon-induced polymerization was carried out at the surface plasmon resonance angle with a laser irradiation input energy of up to $4 \mathrm{~mW}$ and constant flow of monomer/ dye solution at a flow rate of $5 \mu \mathrm{min}^{-1}$. Taking into account the loss of energy, visible by the coupling of the surface plasmon, $35 \%$ of the irradiated energy in case of the one layer film (Fig. $4 \mathrm{~d}$ red) is transmitted into the surface plasmon and thus used for polymerization initiation. After dismantling the flow cell, polymer generation for a thin, $260 \mathrm{~nm}$ thick, film was already visible by eye proving the potential of surface plasmons for polymerization initiation at functionalization of interfaces. Polymer generation was as well supported by infrared spectroscopy clearly showing polymer generation by the disappearing double bond signals at $2770 \mathrm{~cm}^{-1}$ and $1640 \mathrm{~cm}^{-1}$ (Fig. 4e) comparing monomer and polymer infrared spectra. Additionally, the presence of polymer vibrational bands in the absence of monomer vibrational bands for the surface-plasmon induced polymerization in a mesoporous silica film (Fig. 4e) supports polymer functionalization. Performing the surface plasmoninitiated polymerization with a thicker $\sim 560 \mathrm{~nm}$ mesoporous silica film at an angle of $64^{\circ}$, shorter reaction time and under reduced irradiation energy, no or only very small DMAEMA signals were visible in the infrared spectrum (Fig. 4g). This clearly shows the great potential of near-field induced polymerizations for gradual functionalization of mesoporous films applicable in ionic permselectivity control, as well as the huge potential for nanoscale local control on polymer functionalization by benefiting from nanoscale near-field local distribution.

The applicability of visible-light polymer functionalization of mesoporous films to control ionic permselectivity is demonstrated by cyclic voltammetry measurements (Fig. 5). The exemplarily shown cyclic voltammogramms for unmodified mesoporous silica (Fig. 5a) and poly-DMAEMA modified mesoporous silica after plasma treatment and $4^{\prime}, 5^{\prime}$-dibromofluorescein initiated polymerization $(\mathrm{C}=\mathrm{O}$ absorption 0.05 at $1725 \mathrm{~cm}^{-1}$ ) (Fig. 5b) clearly show the influence of polymer functionalization within the mesopores. Due to the positively charged poly-DMAEMA the mesoporous film becomes anionselective and excludes positively charged probe molecules at an acidic $\mathrm{pH}$, whereas the negatively charged molecules are able to enter the pores to a higher concentration as compared to unmodified mesoporous silica films. This is demonstrated by the higher peak current in case of poly-DMAEMA modified films (Fig. 4b) as compared to unmodified silica mesoporous films (Fig. 4a). More results on ionic permselectivity can be found in the ESI. $\dagger$ 

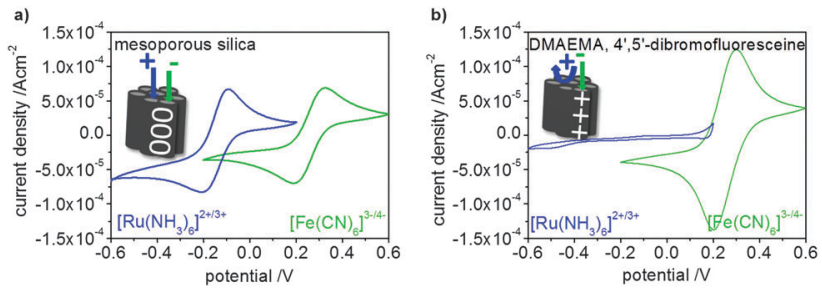

Fig. 5 Cyclic voltammetry $\left(25 \mathrm{mV} \mathrm{s}^{-1}\right)$ results at an acidic $\mathrm{pH}(2-3)$ clearly show the influence of the polymer (here DMAEMA) on ionic permselectivity (b) for a positively charged probe molecule $\left(\left[\mathrm{Ru}\left(\mathrm{NH}_{3}\right)_{6}\right]^{2+13+}\right.$ blue) and a negatively charged probe molecule $\left(\left[\mathrm{Fe}(\mathrm{CN})_{6}\right]^{3-14-}\right.$ green) in comparison to unmodified mesoporous silica (a)

In conclusion, we have developed a mesopore polymer functionalization strategy based on visible light induced radical polymerization which allows mesopore polymer functionalization by near-field modes. Our results suggest polymer functionalization of the inner mesopore walls with lower molecular weights as compared to the polymer generated in solution. Polymer functionalization results in ion permselective behavior governed by the polymer charge. This mesopore functionalization approach is expected to have great potential for local synthetic control with resolution on the nanometer scale and thus especially in the field of nanofluidics and lab on chip devices.

The authors would like to thank the Landesoffensive zur Entwicklung Wissenschaftlich-ökonomischer Exzellenz (LOEWE Soft Control) as well as the Fonds der chemischen Industrie and the Adolf-Messer Stiftung for recognition and financial support of this work. The authors thank Karl Kopp for performing the XPS experiments and Hamid Keshmiri for initial work on mesoporous film preparation on gold surfaces.

\section{References}

1 Z. Nie and E. Kumacheva, Nat. Mater., 2008, 7, 277-290.

2 D. Konkolewicz, K. Schöder, J. Buback, S. Bernhard and K. Matyjaszewski, ACS Macro Lett., 2012, 1, 1219-1223.
3 Z. Nie and E. Kumacheva, Nat. Mater., 2008, 7, 277-290.

4 W. Knoll, A. Kasry, F. Yu, Y. Wang, A. Brunsen and J. Dostalek, J. Nonlinear Opt. Phys. Mater., 2008, 17, 121-129.

5 M. Li, S. K. Cushing and N. Wu, Analyst, 2015, 140, 386-406.

6 C. Deeb, C. Ecoffet, R. Bachelot, J. Plain, A. Bouhelier and O. Soppera, J. Am. Chem. Soc., 2011, 133, 10535-10542.

7 M. Alvarez, A. Best, A. Unger, J. M. Alonso, A. del Campo, M. Schmelzeisen, K. Koynov and M. Kreiter, Adv. Funct. Mater., $2010,20,4265-4272$.

8 J.-P. Fouassier, F. Morlet-Savary, J. Lalevée, X. Allonas and C. Ley, Materials, 2010, 3, 5130-5142.

9 J. Paczkowski and J. Kabatc, Dyes Pigm., 2010, 86, 133-142.

10 O. Valdes-Aguilera, C. P. Pathak, J. Shi, D. Watson and D. C. Neckers, Macromolecules, 1992, 25, 541-547.

11 V. Chegel, M. J. Whitcombe, N. W. Turner and S. A. Piletsky, Biosens. Bioelectron., 2009, 24, 1270-1275.

12 J. M. Belisle, J. P. Correia, P. W. Wiseman, T. E. Kennedy and S. Costantino, Lab Chip, 2008, 8, 2164-2167.

13 J. M. Belisle, D. Kunik and S. Costantino, Lab Chip, 2009, 9, 3580-3585.

14 M. A. Holden and P. S. Cremer, J. Am. Chem. Soc., 2003, 125, 8074-8075.

15 J. C. Scaiano and K. G. Stamplecoskie, J. Phys. Chem. Lett., 2013, 4, $1177-1187$

16 H. Ibn-El-Ahrach, R. Bachelot, G. Lerondel, A. Vial, A. S. Grimault, J. Plain, P. Royer and O. Soppera, J. Microsc., 2008, 229, 421-427.

17 K. H. Dostert, M. Alvarez, K. Koynov, A. del Campo, H. J. Butt and M. Kreiter, Langmuir, 2012, 28, 3699-3703.

18 G. J. A. A. Soler-Illia and O. Azzaroni, Chem. Soc. Rev., 2011, 40, $1107-1150$.

19 S. Alberti, G. J. A. A. Soler-Illia and O. Azzaroni, Chem. Commun., 2015, 51, 6050-6075.

20 C. Sanchez, P. Belleville, M. Popall and L. Nicole, Chem. Soc. Rev., 2011, 40, 696-753.

21 I. Dika, J.-P. Malval, O. Soppera, V. Bardinal, D. Barat, C. Turck, A. Spangenberg and A. Bruyant, Chem. Phys. Lett., 2011, 515, 91-95.

22 L. Silies, H. Didzoleit, C. Hess, B. Stühn and A. Andrieu-Brunsen, Chem. Mater., 2015, 27, 1971-1981.

23 J. E. Spanier and I. P. Herman, Phys. Rev. B: Condens. Matter Mater. Phys., 2000, 61, 10437.

24 A. Andrieu-Brunsen, S. Micoureau, M. Tagliazucchi, I. Szleifer, O. Azzaroni and G. J. A. A. Soler-Illia, Chem. Mater., 2015, 27, 808-821.

25 W. Knoll, Annu. Rev. Phys. Chem., 1998, 49, 569-638.

26 C. J. Brinker, Y. Lu, A. Sellinger and H. Fan, Adv. Mater., 1999, 11, 579-585. 